\title{
Volatile anesthetics and liver injury: a clinical update or what every anesthesiologist should know
}

Jackie L. Martin MD

I $\mathrm{N}$ this issue of the Journal, the topic of postoperative hepatic dysfunction associated with volatile anesthetic agents is revisited. Tung et al. report a case of postoperative liver injury in a patient with normal hepatic function, following an otherwise uncomplicated surgery with desflurane anesthesia. ${ }^{1}$ The patient in this report exhibited many of the characteristics commonly seen in subjects suffering this type of injury. The injury is generally seen three to 28 days after exposure, and is commonly characterized by malaise, fever, jaundice, eosinophilia, marked elevations in serum transaminases and, occasionally, encephalopathy and death. This patient had no fever or eosinophilia, survived and was discharged on postoperative day 21 .

\section{Mechanism of injury}

Once administered, volatile anesthetics, like all drugs, undergo the processes of absorption, distribution, target interaction, metabolism and excretion. However it was once thought that the inhaled anesthetics were chemically inhert. We now know that many of these anesthetic agents undergo significant metabolism and in many cases biotransformation to reactive and potentially toxic intermediates. These metabolic and chemical processes are affected by many factors including age, disease, drug-drug interactions and perhaps most importantly, genetics.

Halothane, isoflurane and desflurane all undergo cytochrome P450 mediated oxidative metabolism to form trifluo acetyl halide metabolites that trifluoroacetylate (TFA) hepatic proteins. ${ }^{2}$ All exposed patients will form these altered tissue proteins (haptens). In sensitized individuals these modified liver proteins, for reasons still unclear, appear to be seen as non-self and induce an immune response which leads to the hepatic injury and sometimes the death of the patient. Thus, anesthetic-induced hepatitis (AIH) is an immune mediated toxicity which occurs in susceptible individuals following exposure to a volatile anesthetic agent. Interestingly, another group of structurally related compounds, the hydrochlorofluorocarbons, have been shown to produce liver injury in humans. ${ }^{3}$ This fact is not surprising, because they are all metabolized in a manner similar to halothane to form TFA-labelled proteins in the liver. ${ }^{4}$ Cross-sensitization between fluorinated inhaled anesthetics has also been documented among halothane, isoflurane, desflurane and enflurane. That is, patients may be sensitized to a volatile anesthetic on one occasion and suffer hepatic injury upon subsequent re-exposure to a different agent. ${ }^{5-7}$

\section{Making the diagnosis}

At the present time AIH remains a diagnosis of exclusion. As in the report by Tung et al. clinicians attempt to exclude all possible causes of acute hepatic injury. Typically, the history and physical examination are reviewed for evidence of exposure to, or ingestion of, toxic chemicals or drugs. Most importantly, any prior history of anesthetic exposure is sought. Viral serologies are tested and a careful review of the perioperative course is undertaken with particular attention to prolonged periods of intraoperative hypotension or hypoxia, exposure to hepatotoxic medications and the transfusion of blood or blood products. Consultants are often utilized and in the end the etiology usually remains undefined. In a previously healthy patient with unexplained postoperative hepatic injury following uncomplicated surgery and anesthesia the volatile anesthetic agent is often then implicated as the causative factor. Immunological assays that detect antibodies against TFA-labelled proteins have been helpful in further implicating inhaled anesthetics as causative agents in liver pathology. 89

From the Department of Anesthesiology and Critical Care Medicine, The Johns Hopkins University School of Medicine, Baltimore, Maryland, USA.

Address correspondence to: Dr. Jackie L. Martin, Department of Anesthesiology and Critical Care Medicine, The Johns Hopkins University School of Medicine, 600 North Wolfe Street, Tower 711 Baltimore, MD 21287-8711, USA. Phone: 410-955-2521; Fax: 410-955-0994; E-mail: jmartin@jhmi.edu 


\section{Risk factors for $\mathrm{AIH}$}

The major risk factor for AIH is a prior history of exposure to fluorinated anesthetic agents. Approximately $95 \%$ of patients will have a history of such exposure. The most common offending agent is halothane. However, injury has also been reported following exposure to enflurane, isoflurane and desflurane. Females are affected more commonly than males, specifically obese, middle-aged women. ${ }^{10}$ Greater than $70 \%$ of affected patients have a history of drug atopy or multiple drug allergies. Chronic ingestion of ethanol or isoniazid are predisposing factors, as both of these drugs induce CYP4502El, the isoform responsible for metabolism of the volatile anesthetic agents.

\section{Occupational exposure and AIH: implications for providers}

Are anesthesia providers at any greater risk than the general surgical population for the development of $\mathrm{AIH}$ as a result of chronic sub-anesthetic exposure to waste anesthetic gases? And, are pediatric anesthesia providers at an even greater risk as the result of mask inductions and the use of uncuffed pediatric endotracheal tubes? Njoku et al. have attempted to address this issue. They reported significantly higher levels of serum autoantibodies associated with anestheticinduced liver injury in pediatric anesthesiologists when compared with general anesthesiologists. ${ }^{11}$ Furthermore, female pediatric anesthesiologists tended to have higher levels of autoantibodies than male pediatric anesthesiologists although illness was reported in only one of 50 female anesthesiologists. These results suggest that autoantibodies, though present in a significant percentage of the study population may not have an important role in AIH.

\section{Management of patients with a history of AIH}

In any patient with a history of unexplained hepatic injury following exposure to inhaled fluorinated anesthetics, avoidance of these agents is the key to a successful operative outcome. A number of anesthetic techniques are available; nitrous-narcotic, total iv anesthesia, regional anesthesia or combined techniques are all suitable alternatives. When anesthetizing patients with a history of AIH it is prudent to remove all anesthetic vaporizers from the anesthesia machine for the duration of the case.

\section{Sevoflurane and AIH}

Sevoflurane is metabolized to hexafluoroisopropanol, inorganic fluoride and formaldehyde. Is does not form the TFA-liver proteins seen following exposure to halothane, isoflurane and desflurane and thus, cannot induce the immune mediated liver injury associated with these agents. Theoretically, patients sensitized to either halothane, isoflurane or desflurane could be safely anesthetized with sevoflurane. However, the package label warns the clinician to avoid sevoflurane in any patient with unexplained liver injury following exposure to any fluorinated inhaled anesthetic.

\section{What's new in inhaled anesthetics?}

The noble gas xenon has anesthetic properties and is of particular interest because it is the only inhert gas which is an anesthetic under normobaric conditions. Xenon was first identified as an anesthetic agent in 1951. ${ }^{12}$ Xenon is a normal constituent in atmospheric air in a concentration no greater than $0.086 \mathrm{ppm}$ and thus, unlike all other inhaled anesthetics, is not an environmental pollutant. The gas cannot be manufactured but is recovered in the process of fractional distillation of liquified air. Xenon is expensive and unlikely to enjoy widespread use due to the expense associated with its extraction from air. If this problem could be overcome, xenon would be the most ideal inhaled anesthetic agent, since xenon (minimum alveolar concentration $=71 \%$ ) is more potent than nitrous oxide, can provide surgical anesthesia in 30\% oxygen and is very insoluble (blood: gas partition coefficient $=0.14)$. Xenon exhibits minimal cardiovascular and hemodynamic side effects, is not known to be metabolized in the liver or kidney, is non-teratogenic and does not trigger malignant hyperthermia in susceptible swine. Xenon is environmentally friendly and does not deplete stratospheric ozone, nor contribute to global warming or the greenhouse effect. The unique combination of analgesia, hypnosis and lack of cardiovascular depression in this one agent makes xenon a very attractive choice for patients with limited cardiovascular reserve.

Over the past decade xenon has been intensely studied in Europe and Japan in a number of clinical trials with very promising results. ${ }^{13-15}$ If anesthetic delivery systems become available which allow for recycling of anesthetic gases, xenon anesthesia may become more economical and thus more readily available in selected patients who might benefit from its lack of adverse systemic effects.

\section{Summary}

The use of any anesthetic agent must be based on specific knowledge of its benefits and risks, how it may produce toxicity and in which patients it may be most safely administered. Clearly, the perfect anesthetic agent does not yet exist and individual patient circumstances will continue to dictate the choice and use of inhaled anes- 
thetics in clinical practice. Reports such as that by Tung et al. remind us of the need for continued vigilance. As ongoing research attempts to uncover emerging toxicities, the clinician is challenged to balance new information with current clinical practices and choose the safest, most effective agents for each patient.

\section{Les anesthésiques volatiles et l'atteinte hépatique : une mise à jour clinique ou ce que tout anesthésiologiste devrait savoir}

Dans le présent numéro du Journal, le sujet de la dysfonction hépatique postopératoire associée aux anesthésiques volatils est revisité. Tung et coll. présentent un patient, dont la fonction hépatique était normale avant l'opération, souffrant d'une lésion hépatique postopératoire après une opération sans complication sous anesthésie au desflurane. ${ }^{1} \mathrm{Il}$ avait de nombreuses caractéristiques communément observées dans ce type d'atteinte. La lésion, qui apparaît généralement de trois à 28 jours après l'exposition, comporte souvent des malaises, de la fièvre, un ictère, de l'éosinophilie, des élévations marquées de transaminases sériques et, à l'occasion, une encéphalopathie et la mort. Ce patient n'a pas eu de fièvre ou d'éosinophilie, il a survécu et a reçu son congé au $21^{\mathrm{e}}$ jour postopératoire.

\section{Mécanisme lésionnel}

Une fois administrés, les anesthésiques volatils subissent, comme tous les médicaments, des processus d'absorption, de distribution, d'interaction ciblée, de métabolisme et d'excrétion. Or, on pensait que les anesthésiques inhalés étaient chimiquement inertes. On sait maintenant qu'ils subissent un métabolisme significatif et, dans de nombreux cas, une biotransformation en intermédiaires réactifs et potentiellement toxiques. Ces processus métaboliques et chimiques sont affectés par de nombreux facteurs dont l'âge, la maladie, les interactions médicamenteuses et, peutêtre le plus important, la génétique.
L'halothane, l'isoflurane et le desflurane subissent tous un métabolisme oxydatif s'exerçant par le cytochrome $\mathrm{P}_{450}$ pour former des métabolites trifluo acétyles haloïdes qui vont produire des protéines hépatiques trifluoroacétylées (TFA). ${ }^{2}$ Ces protéines au tissu modifié (haptènes) se forment chez tous les patients exposés. Chez les individus sensibilisés, ces protéines hépatiques modifiées, pour des raisons encore inconnues, semblent perçues comme non-soi et induisent une réponse immunitaire qui provoque une lésion hépatique et, parfois, la mort du patient. L'hépatite induite par un anesthésique (HIA) représente ainsi une toxicité d'origine immunitaire qui survient chez les individus sensibles après une exposition à un anesthésique volatil. Fait intéressant, on a montré qu'un autre groupe de composés de structure apparentée, les hydrochlorofluorocarbures, produisent des lésions hépatiques chez l'humain. ${ }^{3}$ Ce n'est pas surprenant, car ils sont métabolisés de manière similaire à l'halothane pour former des protéines marquées par des TFA. ${ }^{4}$ La sensibilisation croisée entre les anesthésiques par inhalation fluorés a également été documentée entre l'halothane, l'isoflurane, le desflurane et l'enflurane. Un patient pourrait donc être sensibilisé à un anesthésique volatil lors d'une intervention et souffrir d'une lésion hépatique lors d'une autre exposition à un agent différent. ${ }^{5-7}$

\section{Poser le diagnostic}

Actuellement, l'HIA demeure un diagnostic d'exclusion. Comme le rapportent Tung et coll., les cliniciens tentent d'exclure toutes les causes possibles de lésion hépatique aiguë. En général, les antécédents et l'examen physique sont revus pour vérifier si le patient a été exposé à des produits chimiques ou à des médicaments toxiques ou s'il en a ingérés. Plus important encore, on vérifie toute exposition antérieure à un anesthésique. Les sérologies virales sont testées et une revue attentive de la période périopératoire est entreprise en s'attardant en particulier à des épisodes prolongés d'hypotension ou d'hypoxie peropératoire, à l'exposition à des médicaments hépatotoxiques et à la transfusion de sang ou de produits sanguins. On fait souvent appel à des consultants mais, finalement, la cause demeure habituellement indéfinie. Chez un patient, auparavant en bonne santé, qui présente une atteinte hépatique postopératoire inexpliquée après une opération et une anesthésie sans complications, l'anesthésique volatil est souvent vu comme facteur causal. Les dosages immunologiques qui détectent des anticorps contre des protéines trifluoroacétylées (TFA) ont été utiles dans la mise en cause ultérieure d'anesthésiques par inhalation comme agents responsables de la pathologie du foie. ${ }^{8,9}$ 
Les facteurs de risque d'une HIA

Le principal facteur de risque d'une HIA est une exposition antérieure à des anesthésiques fluorés. Environ $95 \%$ des patients y ont déjà été exposés. L'halothane est le plus en cause. Par ailleurs, une atteinte a aussi été rapportée avec l'enflurane, l'isoflurane et le desflurane. Les femmes sont plus souvent affectées surtout si elles sont obèses et d'âge moyen. ${ }^{10}$ Plus de $70 \%$ des patients touchés ont des antécédents d'atopie médicamenteuse ou d'allergies à de multiples médicaments. L'ingestion chronique d'éthanol ou d'isoniazide sont des facteurs prédisposants, car ces deux substances induisent le CYP4502El, l'isoforme responsable du métabolisme des anesthésiques volatils.

\section{L'exposition professionnelle et l'HIA : les implica- tions pour les prestateurs d'anesthésie}

Les prestateurs d'anesthésie sont-ils plus à risque que la population chirurgicale générale d'avoir une HIA résultant d'une exposition subanesthésique de longue durée aux gaz résiduaires ? Les anesthésistes pédiatriques sont-ils encore plus à risque, conséquence des inductions au masque et de l'usage de tubes endotrachéaux pédiatriques sans ballonnets ? Njoku et coll. ont tenté de répondre. Ils ont noté des hausses significatives des niveaux sériques d'autoanticorps associés à une lésion hépatique induite par anesthésiques chez des anesthésiologistes pédiatriques comparés à des anesthésiologistes généraux. ${ }^{11}$ De plus, les femmes anesthésiologistes en pédiatrie ont généralement des niveaux plus élevés d'autoanticorps que leurs collègues masculins quoique la maladie n'ait été citée que chez une parmi 50 femmes anesthésiologistes. Ce qui montre que les autoanticorps, même présents selon un pourcentage significatif dans la population étudiée, n'ont peut-être pas un rôle important dans l'HIA.

\section{Prise en charge des patients aux antécédents d'HIA}

Chez tout patient qui présente une atteinte hépatique inexpliquée après une exposition aux anesthésiques fluorés inhalés, le fait d'éviter ces agents est la clé du succès chirurgical. Un certain nombre de techniques anesthésiques est possible : une combinaison de narcotiques et de protoxyde d'azote, une anesthésie exclusivement $i v$, une anesthésie régionale ou des techniques combinées. Quand on anesthésie des patients qui ont des antécédents d'HIA, il est prudent de retirer tous les évaporateurs de l'appareil d'anesthésie pour la durée de l'opération.

\section{Le sévoflurane et l'HIA}

Le sévoflurane est métabolisé en hexafluoro-isopropanol, du fluor inorganique et du formaldéhyde. Il ne forme pas les protéines TFA qu'on voit après l'exposition à l'halothane, à l'isoflurane et au desflurane et, donc, ne peut induire la lésion hépatique d'origine immunologique associée à ces médicaments. Théoriquement, les patients sensibilisés à l'halothane, à l'isoflurane ou au desflurane pourraient être anesthésiés sans danger avec le sévoflurane. Mais, le dépliant commercial avertit le clinicien d'éviter le sévoflurane chez tout patient atteint d'une lésion hépatique inexpliquée survenue à la suite d'une exposition à tout anesthésique fluoré inhalé.

\section{Quoi de neuf concernant les anesthésiques inhalés ?}

Le gaz rare xénon a des propriétés anesthésiques et est particulièrement intéressant, car c'est le seul gaz inerte qui soit anesthésique dans des conditions normobares. Le xénon a été identifié pour la première fois comme anesthésique en 1951. ${ }^{12}$ Il est un constituant normal de l'atmosphère selon une concentration aussi faible que $0,086 \mathrm{ppm}$ et, par conséquent, n'est pas un polluant de l'environnement comme les tous les autres anesthésiques inhalés. Le gaz ne peut être fabriqué, mais il est récupéré dans le processus de distillation fractionnée de l'air liquide. Le xénon est cher et son usage risque peu de se répandre à cause du coût d'extraction. Si on pouvait surmonter ce problème, le xénon pourrait être l'anesthésique d'inhalation idéal, puisqu'il (concentration alvéolaire minimale $=71 \%$ ) est plus puissant que le protoxyde d'azote (sang : coefficient de partage des gaz $=0,14)$. Le xénon a des effets secondaires cardiovasculaires et hémodynamiques minimaux, n'est pas métabolisé dans le foie ou les reins, n'est pas tératogène et ne provoque pas d'hyperthermie maligne chez des porcs sensibles. Le xénon est sans danger pour l'environnement et ne réduit pas l'ozone stratosphérique, ne contribue pas au réchauffement global ou à l'effet de serre. La combinaison unique d'analgésie, d'hypnose et d'absence de dépression cardiovasculaire chez ce seul agent fait du xénon un choix très intéressant pour les patients dont la réserve cardiovasculaire est limitée.

Pendant la dernière décennie, on a beaucoup étudié le xénon en Europe et au Japon dans nombre d'essais cliniques aux résultats très prometteurs. ${ }^{13-15} \mathrm{Si}$ des appareils d'anesthésie permettant de recycler les gaz sont mis au point, l'anesthésie au xénon pourrait être plus économique et, donc, choisie plus volontiers pour des patients sélectionnés qui pourraient bénéficier de l'absence de ses effets systémiques indésirables.

\section{Conclusion}

L'usage de tout anesthésique doit reposer sur la connaissance spécifique de ses avantages et de ses risques, 
de la façon qu'il peut produire de la toxicité et des patients chez qui il peut être administré sans danger. Certes, l'anesthésique parfait n'existe pas encore et les particularités individuelles des patients guideront encore le choix et l'usage cliniques des anesthésiques par inhalation. Des articles comme celui de Tung et coll. rappellent la nécessité d'être toujours vigilant. Au moment où la recherche en cours tente de découvrir de nouvelles toxicités, le clinicien fait face au défi d'équilibrer les informations récentes et les habitudes cliniques courantes et de choisir l'anesthésique le plus sûr et le plus efficace pour chaque patient.

\section{References}

I Tung D, Yoshida EM, Wang CS, Steinbrecher UP. Severe desflurane hepatotoxicity after colon surgery in an elderly patient. Can J Anesth 2005; 52: 133-6.

2 Njoku D, Laster MJ, Gong DH, et al. Biotransformation of halothane, enflurane, isoflurane, and desflurane to trifluoroacetylated liver proteins: association between protein acylation and hepatic injury. Anesth Analg 1997; 84: 173-8.

3 Hoet P, Graf ML, Bourdi M, et al. Epidemic of liver disease caused by hydrochlorofluorocarbons used as ozone-sparing substitutes of chlorofluorocarbons. Lancet 1997; 350: 556-9.

4 Harris JW, Pobl LR, Martin JL, Anders MW. Tissue acylation by the chlorofluorocarbon substitute 2,2dichloro-1,1,1-trichloroethane. Proc Natl Acad Sci USA 1991; 88: 1407-10.

5 Sadove MS, Kim SI. Hepatitis after use of two different fluorinated anesthetic agents. Anesth Analg 1974; 53: $336-40$.

6 Sigurdsson J, Hreidarsson AB, Thejodleifsson B. Enflurane hepatitis. A report of a case with a previous history of halothane hepatitis. Acta Anaesthesiol Scand 1985; 29: 495-6.

7 Christ DD, Kenna JG, Kammerer W, Satoh H, Pobl LR. Enflurane metabolism produces covalently bound liver adducts recognized by antibodies from patients with halothane hepatitis. Anesthesiology 1988; 69: 833-8.

8 Martin JL, Kenna JG, Pohl LR. Antibody assays for the detection of patients sensitized to halothane. Anesth Analg 1990; 70: 154-9.

9 Martin JL, Keegan MT, Vasdev GM, et al. Fatal hepatitis associated with isoflurane exposure and CYP2A6 autoantibodies. Anesthesiology 2001; 95: 551-3.

10 Cousins MJ, Plummer JL, Hall PD. Risk factors for halothane hepatitis. Aust N Z Surg 1989; 59: 5-14.

11 Njoku DB, Greenberg RS, Bourdi M, et al. Autoantibodies associated with volatile anesthetic hepatitis found in the sera of a large cohort of pediatric anesthesiologists. Anesth Analg 2002; 94: 243-9.
12 Cullen SC, Gross EG. The anesthetic properties of xenon in animals and human beings, with additional observations on krypton. Science 1951; 113: 580-2.

13 Lachmann B, Armbruster S, Schairer W, et al. Safety and efficacy of xenon in routine use as an inhalational anaesthetic. Lancet 1990; 335: 1413-5.

14 Luttropp HH, Thomasson R, Dahm S, Persson J, Wernfr $O$. Clinical experience with minimal xenon anesthesia. Acta Anaesthesiol Scand 1994; 38: 121-5.

15 Rossaint R, Reyle-Hahn M, Schulte Am Esch J, et al. Multicenter randomized comparison of the efficacy and safety of xenon and isoflurane in patients undergoing elective surgery. Anesthesiology 2003; 98: 6-13. 